\title{
Nursing for Renal Replacement Therapies in the Intensive Care Unit: Historical, Educational, and Protocol Review
}

\author{
lan Baldwin Nigel Fealy \\ Department of Intensive Care, Austin Hospital, Melbourne, Vic., Australia
}

\section{Key Words}

Continuous renal replacement therapy $\cdot$ Nursing $\cdot$ Intensive care unit $\cdot$ Haemofiltration $\cdot$ Acute renal failure

\begin{abstract}
Nurses have made a significant contribution to the development and application of dialysis in the 1950s and continuous renal replacement therapies (CRRT) in the Intensive Care Unit (ICU) setting from the 1980s. Any treatment requires patient and machine-circuit preparation, connection of the extracorporeal circuit $(E C)$ to the patient vascular access catheter and regular tasks to maintain a treatment in progress. During treatment, nurses prepare fluids, adjust fluid settings to provide fluid balance, prepare electrolyte additives, monitor acid base and electrolyte levels, monitor patient and machine 'vital signs', and then when necessary diagnose circuit clotting and perform a disconnection of the EC from the patient. All of these aspects of CRRT nursing are essential to a suitable nursing policy or protocol. This paper provides a clinical review for this every day sequence when using CRRT in the ICU setting.

Copyright $\odot 2009$ S. Karger AG, Basel
\end{abstract}

\section{Introduction}

Despite complex physiology, human kidney function can be replaced with an artificial process which can sustain life for many years.

The kidneys have an important interrelationship with other key body organs such as the brain, heart, liver and lungs that are dependent on their function. With high levels of 'toxins' in acute renal failure (ARF) these organs also become dysfunctional $[1,2]$.

However, as these interrelationships continue to be further described, the use of artificial kidney techniques for the management of ARF in critical illness is primarily focussed on replacing the essential kidney functions. They are fluid balance, solute removal, acid base and electrolyte control [3-6].

This paper provides a clinical protocol approach to the use of renal replacement therapy (RRT) in the ICU for nursing and includes: preparation of the RRT machine and patient, connection of the circuit, management and trouble-shooting during treatment, stopping treatment and disconnection of the circuit.

This paper also provides an education focus beginning with a brief historical review for the development of the artificial kidney and basic science concepts for dialysis and nursing. 

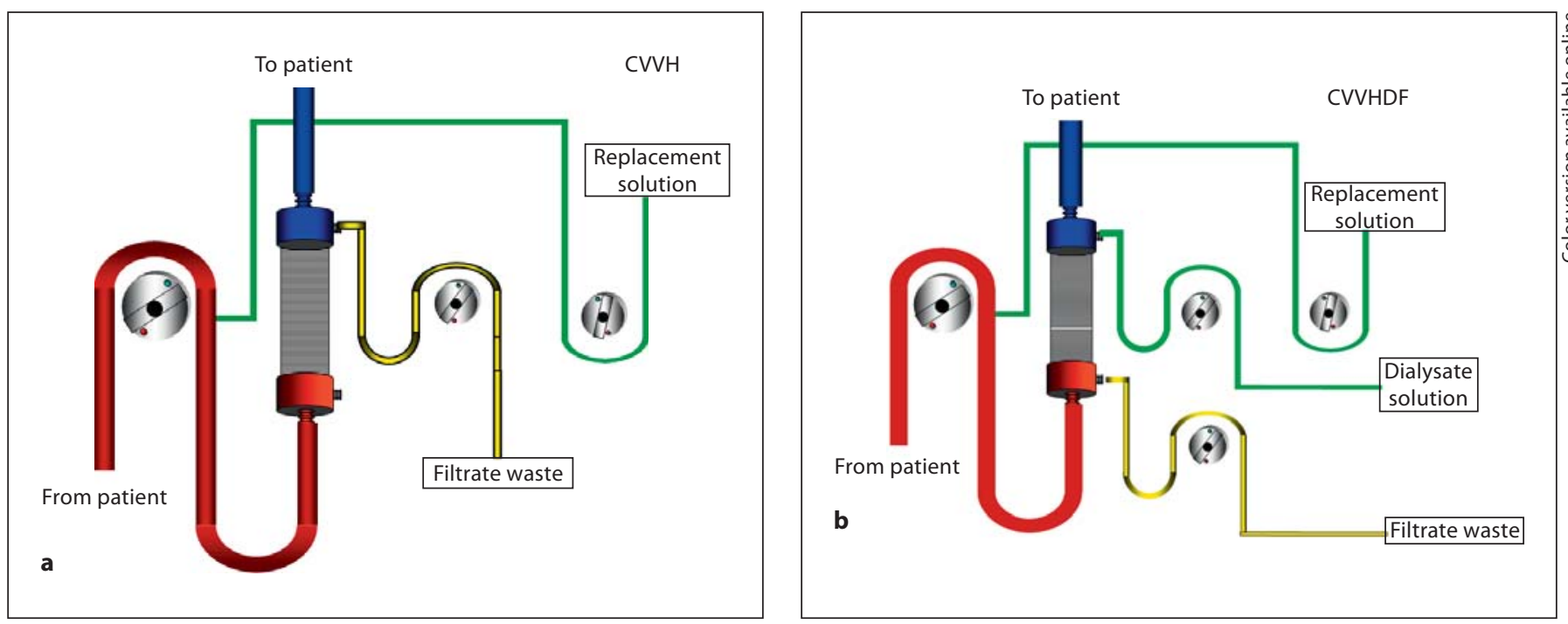

Fig. 1. a Convective clearance haemofiltration or CVVH. b Diffusive and convective clearance haemodialfiltration or CVVHDF.

\section{Historical Review and Key Concepts of Dialysis}

The term 'dialysis' refers to the filtering of blood through a membrane [7]. The first use of the term was by a Scottish chemist, Thomas Graham in 1854, who used 'dialysis' to describe the transport of solutes through an ox bladder and drew attention to the concept of a membrane for solute removal from fluid - diffusion [7]. The first human dialysis was carried out by a German physician, George Haas, in the late 1920s performing 6 treatments on 6 patients [8]. In the 1920s and 1930s, synthetic polymer chemistry developed cellulose acetate, which was used extensively in the food packaging and sausage industry. This material was later used for dialysis membranes [9]. The development of the anti-clotting drug, heparin, made dialysis possible by preventing blood clotting when outside the body in cellulose and plastic tubing [10].

The technology was further developed during the Second World War by a Dutch physician named Willem Kolff. The Kolff rotating drum kidney used a cellulose tube wound around a wooden skeleton built as a large drum-styled cage. The drum with the blood-filled cellulose tubing wound around it was immersed in a bath of weak salt solution and, as blood passed through the rotating drum cellulose tubing, waste exchange occurred $[10$, $11]$.

In the 1950s Fredrik Kiil, in Norway, developed the parallel plate dialyser made of a new cellulose, cupro- phane. This required a pump to push the blood through the membrane and return it to the patient [12]. The hollow fibre membrane dialyser was later developed by a physician, Richard Stewart, in association with the United States company Dow Chemical Corporation in the early 1960s with first use of the device in 1967. The membrane was made of cellulose acetate with 11,000 fibers in a bundle providing a surface area of $1 \mathrm{~m}^{2}$ [8]. The same design concept is used today with new fiber development using polymer such as polysulfone and other artificial synthetic chemical structures better imitating the nephron glomerulus and the ability to transfer wastes and plasma water. This form of medical treatment and associated nursing management is now commonly known as haemodialysis. The major components are essentially the same with the key component being the membrane also called the 'artificial kidney' or 'dialyser'. Over the last 50 years, industrial and scientific developments such as plastics moulding and electronics have made current dialysis techniques safe and effective [13].

\section{Principles of Renal Replacement Therapy}

The principles of RRT are to draw blood from the body via a venous access catheter placed in a large vein, e.g. jugular or femoral vein. A pump sucks the blood from the patient and then pushes it into the chief processing unit: the membrane/dialyser. This allows either the removal of 
solutes and wastes by plasma water removal - convection or exchange of solutes by concentration difference or diffusion [14]. Convection is essentially a filtering process where plasma water and anything dissolved in the water is removed together and discarded. Commonly referred to as haemofiltration or continuous veno-venous haemofiltration $[15,16](\mathrm{CVVH})$; figure 1a is a circuit diagram for this technique.

Alternatively, blood can be exposed to a dialysate solution in the membrane, and diffusion takes place exchanging solutes without plasma water removal. Both mechanisms can be performed together and is termed haemodiafiltration or continuous veno-venous haemodiafiltration [14-16] (CVVHDF) (fig. 1b).

When plasma water removal is done during convective removal, a suitable electrolyte solution must be given to replace this [17]. As the replacement solution has no toxic wastes, the net result is waste removal. Where a negative fluid balance is required, less replacement solution is given compared to that removed.

Blood outside the body will begin to clot unless the coagulation mechanisms are blocked or influenced to delay clotting. Anticoagulants such as heparin or sodium citrate can be given, dilution of the blood with replacement solutions, and maintenance of an unobstructed blood flow around the extracorporeal circuit are very important to achieve this [18].

Although a machine provides the therapy, without correct, skilled, efficient and safe use, the treatment may fail and or patient safety be at risk. Nurses are the vital link making this achievable in a clinical patient care context.

\section{Brief Historical Perspectives of Dialysis Nursing}

Nurses have made a contribution to the safety and efficiency of dialysis, with the first meeting of nurses to discuss this special interest and skills taking place in the United States in 1966 at the Boston Peter Bent Brigham Hospital [19]. The convenor of this meeting, Barbara Coleman $\mathrm{RN}$, is recognised as the first dialysis nurse publishing a treatment protocol for dialysis using the rotating drum machine in 1952 [19, 20]. Nurses were reported to be involved in the very first treatments performed by Kolff in the 1940s and played an important role as a number of centres attempted their first dialysis procedure.

Hoffart [19] describes many of these initial patients being like 'guinea pigs'. In addition to preparing the machine, mixing fluids, monitoring vital signs, and per- forming documentation, nurses made the patient comfortable and provided personal care. This was often in a setting with a 'side show' atmosphere, as many interested health and scientific groups watched on during the treatment promoted at the time as an 'eighth wonder of the world'. Hoffart [19] reminds us that as many patients died during such treatments, nurses held an important responsibility to ensure such events held some dignity for the patient and their family. History has seen dialysis move from research laboratories into specialist hospitals, non-hospital dialysis clinics, patient homes and, with a collaborative approach, into the ICU for a further type of dialysis required in the critically ill $[6,21,22]$. Nurses have been a central key to all these developments. These are foundations of dialysis nursing with many of them continuing unchanged in principle today as dialysis treatment is a specialist field of knowledge and skill for nurses both in the outpatient and ICU setting [23, 24].

For CRRT to be performed safely and with efficiency, a nursing protocol or policy can be developed by key staff involved. This document is particularly useful for new staff and learners. The following provides a guide for everyday use of CRRT and is the basis for a nursing protocol describing how to prepare the patient and machine, connect the EC and manage a treatment. Then, when necessary cease treatment and disconnect the EC.

\section{Patient and Machine, Preparation}

\section{Patient Position}

Prior to connection patients should be positioned appropriately. Critically ill patients should be positioned in a supine position and remain in bed for the connection procedure. However, less ill patients may also be connected to CRRT when out of bed in a suitable reclining chair if cardiovascular and respiratory acuity permits. Once treatment has been established and both machine and patient demonstrate stability, the patient can be repositioned for pressure area care and physiotherapy. To avoid kinking of the access catheter, when transferring a patient from the chair to a bed (using a 'slide board' or similar device) pausing fluids exchange and slowing the blood pump speed to $100 \mathrm{ml} / \mathrm{min}$ can prevent high circuit pressures and alarm events stopping the blood pump during the transfer procedure.

\section{Access Catheter}

The vascular access catheter is the most important component of a successful treatment $[25,26]$. A check of 
this catheter is very important prior to connection. If there have been frequent, premature ( $<4 \mathrm{~h}$ function) filter failures, the catheter should be examined as the potential cause $[18,27]$. Function of the access device may be determined by the ability to withdraw blood from the 'arterial' limb and flushing of the 'venous limb' with a saline syringe. Any failure in aspiration or high resistance to flushing of the device may mandate a new catheter before attempting connection.

\section{Monitoring}

For critically ill patients monitoring of haemodynamic status before and during connection is an essential element in the safe provision of establishing an extracorporeal circuit. Continuous arterial blood pressure (ABP), commonly associated with ICU care, is monitored closely on initiation of the extracorporeal circuit to detect any hypotension.

\section{Connecting to Circuit}

The connection procedure should be done as a sterile procedure and requires two nurses. The primary nurse's responsibility is to maintain a sterile field and connect the 'arterial' and 'venous' limbs of the extracorporeal circuit to the access device after soaking and cleaning the catheter ends with chlorhexidine 70\% alcohol solution. The secondary nurse will 'hand off' the circuit lines to the primary nurse and interact with the CRRT machine to start the blood pump.

\section{Machine}

It may be an advantage to set up and prime the machine away from the bedside. Novices to the preparation of a CRRT machine may benefit from more focused attention where interruption from other staff and patient responsibilities can interfere with the learning process. This may also facilitate optimal instruction and training from clinical educators to guide the novice nurse through the initial setup and priming process. More experienced nurses who are proficient or experts and skilled in machine and circuit preparation can undertake the priming process with interruption and attend to patient care simultaneously $[21,23]$.

\section{Prime Solution}

Most machines require a priming solution to fill the extracorporeal circuit and dialyser removing all air. Commonly, saline is utilised to perform this procedure and is useful as a 're-circulation' fluid prior to connection. This solution is also administered to the patient when starting the treatment and drawing patient blood into the EC. Substitution fluids used for the treatment may also be used; however, bicarbonate-buffered solutions yield gas bubbles especially when heated and can be difficult to expel during priming.

\section{Pre-Checklist}

After priming is complete, it may be beneficial to undertake a pre-treatment checklist to confirm machine alarm parameters, treatment settings for fluids and fluid loss, venous chamber blood level, excessive circuit bubbles, mains power is on uninterruptible power supply (UPS), normal saline (e.g. $500 \mathrm{ml}$ ) may be connected to the circuit for emergency return of blood to the patient, and anticoagulant infusion dose is correct.

\section{Re-Circulation}

If machine setup and the priming process is performed in advance of the connection procedure, the saline-filled circuit may be 're-circulated'. This may be an option on some machines software, or requires 'treatment mode' to be activated. With both arterial and venous limbs connected (spiked) into a normal saline bag, the blood pump can be commenced and maintained at a low pump speed $(50 \mathrm{ml} / \mathrm{min})$. If heparin coating of the circuit and dialyser is desired, then heparin can be added to the recirculation fluid to improve distribution of the anticoagulantoptimising effect $[28,29]$. As no data is available to suggest otherwise, a primed circuit may be re-circulated up to $48 \mathrm{~h}$ prior to connection to a patient if circuit integrity is maintained.

\section{Starting Blood Flow and Treatment}

Establishing the extracorporeal circuit for renal replacement therapy in the critically ill patient can be associated with haemodynamic instability. Air embolus, collapse of venous return with rapid initial blood flow rate, no concurrent connection of the venous return limb of the circuit, anaphylactoid patient response to the membrane and blood exposure, use of heated and or albumin prime solution, concurrent uncontrolled cardiac arrhythmias such as VT or rapid AF and the shocked fluid depleted patient are all potential causes $[24,30]$.

If patient haemodynamic stability can be achieved before starting treatment, this should be attempted. In all cases, however, the blood pump must be started slowly and increased in speed with increments of $20-50 \mathrm{ml} / \mathrm{min}$ until full operating blood flow is achieved; commonly $200 \mathrm{ml} / \mathrm{min}$. This may take around $5 \mathrm{~min}$, and only then should the exchange of fluids be activated effectively 


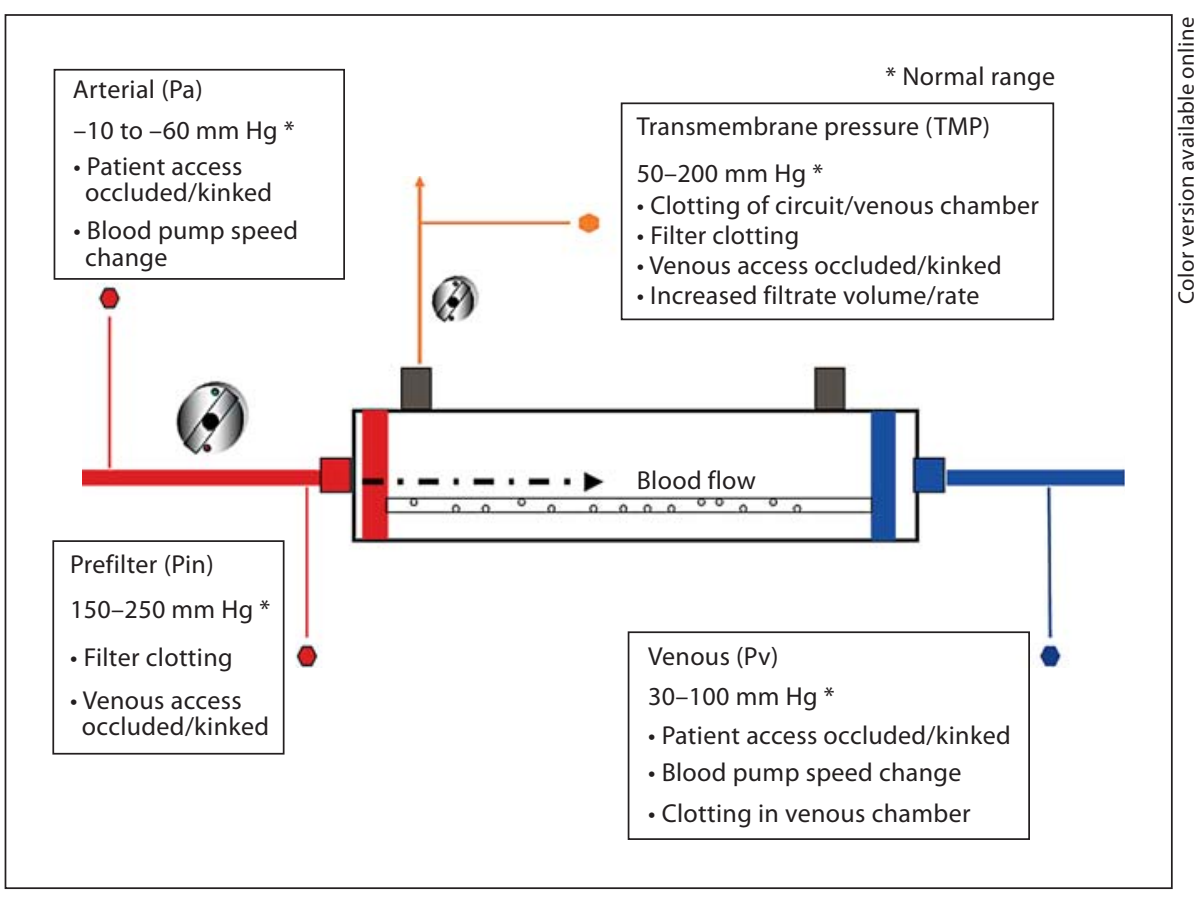

Fig. 2. Circuit diagram indicating common pressure readings, normal ranges and causes of these alarms.

starting the treatment. Starting fluid removal at low blood flow will cause a filtration fraction mismatch and an alarm event on some machines, and in most cases, a negative fluid loss is not helpful until the patient is stable after EC connection.

\section{Treatment in Progress}

Stay with the Patient for 15 Minutes after Starting. Check/re-check treatment and fluid settings, re-check circuit connections. Monitor patient for any change in vital signs.

Machine-Measured 'Vital Signs'. All CRRT machine measure pressures from the circuit to reflect correct function. It may be useful to document some of these pressures on an hourly basis along with other patient vital signs, e.g. transmembrane pressure (TMP) or pressure IN (P-IN) reflecting membrane clotting. However, as alarms can be set to detect any aberration in these pressures, most nurses do not document the values and prefer to review them from a screen display. Common circuit pressures and normal ranges are indicated in figure 2.

Fluids Preparations and Bag Changes. Commercial solutions for CRRT are prepared in 5-litre bags. This provides a physiological electrolyte solution suitable for both dialysate fluid and substitution fluid [17]. Potassium additive is often required; however, hyperkalaemia may be present initially. For this reason, the fluids are of 0 $5 \mathrm{mmol} / \mathrm{l}$ potassium concentration when manufactured. It is common to add $20-15 \mathrm{mmol} / \mathrm{l}$ of potassium making a concentration of $4.0 \mathrm{mmol} / \mathrm{l}$ for continuous use.

Additives can be done ahead of time, and where bicarbonate solutions are used, the bags may be stored with electrolyte and bicarbonate solution in separate compartments. A plastic plug usually separates the two fluids and requires breakage to enable mixing. This can be done just prior to hanging the bag, but can be forgotten and a treatment with no buffer results. A sticker placed on the outside of the bag indicating 'mixed' or 'bicarb added' may minimise this error.

Fluid Balance and Prescription. The nurse managing the treatment is effectively responsible for ensuring the settings are achieving the physician orders. The orders need to be clear to the nurse, in both a bedside case - discussion and in writing as mistakes can occur. Intensive care units commonly have CRRT order sheets at the bedside for this [31,32]. The order sheets usually make clear that treatment goals (usually a loss - fluid removal) are achieved from 'machine' settings achieving negative fluid loss, but also consider patient fluids inputs. For example, a treatment may be set for a $200-\mathrm{ml} / \mathrm{h}$ fluid removal where the intravenous inputs are also $200 \mathrm{ml} / \mathrm{h}$; this is considered patient 'even' or no loss. 
Fig. 3. Infomed HF 440 CRRT machine (Infomed, Geneva, Switzerland) incorporating bottle collection of waste fluids. Small electric fluids pump mounted to machine frame and drain line direct to waste developed by TekMed, Melbourne, Vic., Australia.

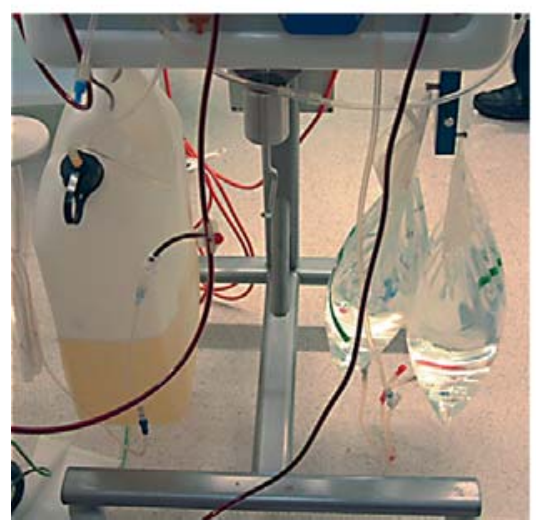

Collection bottle in use, front view

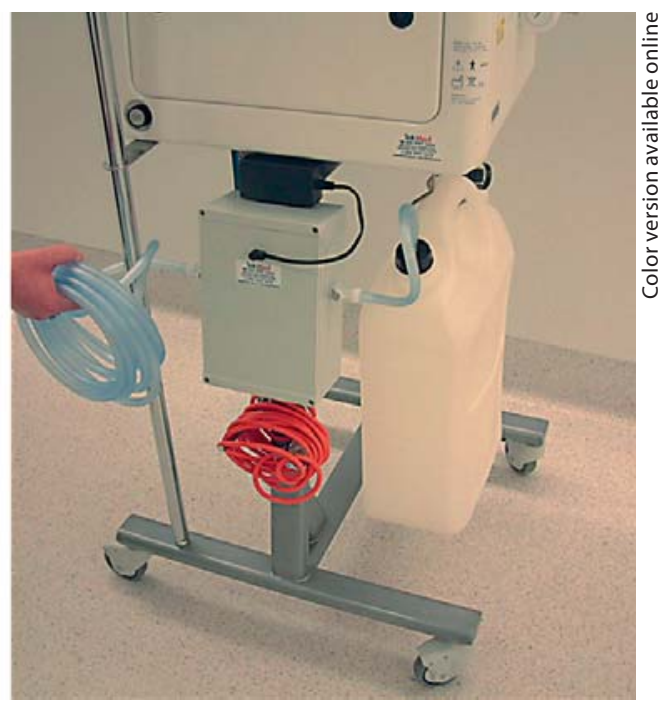

Rear view of 25-litre collection bottle, waste fluids pump and hose

\section{Electrolyte and Acid Base Monitoring}

Routine care for patients in the ICU mandates frequent electrolyte monitoring in association with arterial blood gas and acid base analysis. Of clinical importance are the potassium results for the maintenance of adequate additive to commercial CRRT solutions. Blood gases with acid base and electrolyte values measured 4-6 hourly is adequate for patients treated with CRRT. More frequent measurements may be done as a reflection of the patient respiratory status and critical illness, although not necessary for CRRT, are helpful monitoring.

\section{Documentation of Treatment Hours}

Recording sequential hours (1, 2, 3, 4, etc.) of circuit function from start of each treatment on the bedside charts is useful information monitoring treatment. This reveals both the current status and success of each circuit in use with respect to effective treatment time before clotting. In addition, the 'off time' is easily identified as there will be time gaps reflecting 'no treatment' [33]. When the corresponding anticoagulation type and dose is documented with the successive circuit hour, a link between anticoagulation and total circuit function can be easily determined.

\section{Waste Management}

Removal and disposal of spent dialysate or ultrafiltrate waste can be a burden for nurses. Machine and circuit design often collects the waste into a large plastic bag with a 5- to 10-litre capacity. These bags when full must be disposed of to a suitable sewer drain or bedpan room outlet requiring staff to carry the waste bags to the room. Although the waste fluid does not contain bacteria, such body fluids are considered a biohazard if spilt. It is ideal if the waste can be drained to a waste outlet directly and continuously during treatment, or pumped to a drain after a period of collection. When fluid balance is determined by machine scales assessing a change in the weight (increasing) of waste bags, drainage during treatment is not possible. However, to eliminate handling of full waste bags, the waste can be collected in a bottle placed on the machine scales, and when full, pump this waste via a tubing line to a drain outlet near the patient's bed (fig. 3). A small pump is incorporated into the machine frame (Infomed HF 440 CRRT machine, Infomed, Geneva, Switzerland, in association with TekMed, Melbourne, Australia) to do this and the emptying procedure takes approximately $4 \mathrm{~min}$. When complete, the treatment is continued with the frequency of the procedure related to the capacity of the collection bottle and/or where the 'waste full' alarm (e.g. $20 \mathrm{~kg}=20$ litres) is set. Once per nursing shift is usual. Where suitable drain outlets are available near to an ICU bed $(<10 \mathrm{M})$, this eliminates the need to carry waste fluids to a room for drainage and reduces use of plastic bags as the 25 -litre plastic bottle is re-used after cleaning between patients.

\section{Predicting Filter and Circuit Clotting}

Predicting filter clotting is a useful nursing aspect of CRRT [34]. It is desirable to return blood from the circuit 
to the patient before clotting and obstruction of the circuit preventing this. In addition, is it preferable to cease a treatment when nurses are available for the procedure rather than during staff breaks or at shift handover time. Therefore, indicators that circuit failure is imminent are useful to cease treatment at a convenient time and return the patient blood. Some nurses believe they can predict filter clotting by looking at the membrane and or venous chamber for visible clot after a saline bolus-flush (200$300 \mathrm{ml}$ ) to reveal clot development. While there is no data to support this method, pressure measurements from the EC are more reliable [35-37]. Circuit pressure measurements are influenced by treatment modality, blood pump speed and fluid settings; however, the following is a suggested guide for adult patients with a blood flow rate of $200 \mathrm{ml} / \mathrm{min}$. They are: TMP > $250 \mathrm{~mm} \mathrm{Hg}$, P-IN >200 $\mathrm{mm} \mathrm{Hg}$ and P-transfilter gradient $>26 \mathrm{~mm} \mathrm{Hg}$ (difference between pre- and post-filter pressure $=\mathrm{P}$-IN minus P-Venous) $[36,37]$.

\section{Ending Treatment}

Disconnection from the CRRT machine and circuit may be temporary for diagnostics such as CT scans or operative procedures, or where treatment is stopped upon some renal recovery.

The aim of the disconnection procedure is to return to the patient the volume of blood in the extracorporeal circuit (approximately $120 \mathrm{ml}$ ). To commence the procedure, any anticoagulation being infused into the circuit should be ceased. Treatment (replacement, dialysate and ultrafiltrate pumps) should be ceased and any volumes recorded on the fluid balance chart. On most current machines this would be part of a 'recirculation' or 'end treatment' option in the operating screen software. On screen, instructions to perform the disconnection procedure are usually well presented and describe how to return blood to the patient. The following is a suitable description for this.

\section{Procedure}

Disconnect the 'arterial' limb from the vascular access and 'spike' a saline bag or connect a saline flush bag to a port of the arterial limb of the circuit. Clamp the outflow limb of the access catheter or arterial blood line adjacent to the access catheter. Open the saline flush line immediately prior to this. At a reduced blood pump speed $(100 \mathrm{ml} / \mathrm{min})$ flush circuit blood back into the patient for $1.0-1.5 \mathrm{~min}$ (i.e. $100-150 \mathrm{ml}$ of saline), stop the blood pump. All lines including access, bloodlines and flush system should now be clamped to avoid blood ex- posure. Saline flush should be recorded on fluid balance chart.

\section{Disconnection of Lines}

If treatment is ceased for procedure or scan, recirculation or similar option should be selected on machine. On screen software should prompt users to hand over 'arterial' and 'venous' ends to an assistant using aseptic technique. The assistant then connects both lines to a 1-litre normal saline bag using dialysis 'spikes' and the blood pump recommenced at $\sim 50-100 \mathrm{ml} / \mathrm{min}$ to recirculate solution through the circuit and dialyzer.

\section{Vascular Access}

Once bloodlines have been disconnected, using a sterile technique, the vascular access lumens should be flushed with normal saline (approximately $10 \mathrm{ml}$ ) and device assessed for patency. Anticoagulant 'locking' may be used to prevent access catheter clotting [23, 24]. Check volumes required to fill the lumen deadspace, instil this volume with anticoagulant (e.g. heparin, citrate) and clamp lumens [38]. The lumens should then be capped with a sterile cap. The vascular access site should then be assessed for signs of infection and dressing inspected to maintain occlusive covering. Documentation for this procedure should be recorded in the nursing shift notes and or bedside charts.

\section{Conclusion}

This review has provided an education focus for the history of dialysis nursing and basic concepts of how CRRT works. This information sets the basis for a policy document necessary in any clinical area where CRRT is applied. All the necessary and sequential steps for a treatment are described. They are patient and machine - circuit preparation, connection of the extracorporeal circuit to the patient vascular access catheter and nursing management of a treatment in progress. This management included fluids preparation, adjustment of fluid settings and electrolyte additives to provide fluid balance, acid base and electrolyte control, and monitoring of patient and machine 'vital signs'. The final step discussed is the diagnosis of 'circuit clotting' and how to perform a disconnection of the circuit and machine from the patient. 


\section{References}

1 Kellum JA, Hoste EA: Acute renal failure in the critically ill: impact on morbidity and mortality. Contrib Nephrol. Basel, Karger, 2004, vol 144, pp 1-11.

2 Chien-Chu C, Landon KS, Rabb H: Mechanisms underlying combined acute renal failure and acute lung injury in the intensive care unit. Contrib Nephrol. Basel, Karger, 2004, vol 144, pp 53-62.

3 Burchardi H: History and development of continuous renal replacement techniques. Kidney Int 1998;53(suppl 66):S120-S124.

4 Bellomo R, Ronco C: Continuous renal replacement therapy in the intensive care unit. Intens Care Med 1999;25:781-789.

5 Bellomo R, Ronco C: Continuous versus intermittent renal replacement therapy in the intensive care unit. Kidney Int 1998;53(suppl 66):S125-S128.

6 Ronco C, Cruz D, Bellomo R: Continuous renal replacement in critical illness. Contrib Nephrol. Basel, Karger, 2007, vol 156, pp 309-319.

7 Smith T, Thomas N: The history of dialysis and transplantation, chap 1 ; in Thomas $\mathrm{N}$ (ed): Renal Nursing, ed 2. Sydney, Elsevier Science Limited, 2002.

8 Gottschalk C, Fellner S: History of the science of dialysis. Am J Nephrol 1997;17:289298.

D9 McBride P: Industry's contributions to the development of renal care. ANNA J 1989;16: 217-226.

10 Cameron JS: Practical haemodialysis began with cellophane and heparin: the crucial role of William Thalhimer (1884-1961). Nephrol Dial Transplant 2000;15:1086-1091.

$\checkmark 11$ Kolff WJ: Dialysis in the treatment of uremia. Arch Intern Med 1954;94:142-147.

$\checkmark 12$ Cole J, Pollard T, Murray J: Studies on the modified polypropylene Kiil dialyser. Trans Am Soc Artif Intern Org 1963;9:67-70.

13 Parker TF: Technical advances in haemodialysis therapy. Semin Dialy 2000;13:327377.

$\checkmark 14$ Ronco C, Bellomo R: Basic mechanisms and definitions for continuous renal replacement therapies. Int J Artif Org 1996;19:95-99.
15 Ronco C, Bellomo R: Continuous renal replacement therapy: evolution in technology and current nomenclature. Kidney Int 1998; 66(suppl):S160-S164.

16 D'Intini V, Ronco C, Bonello M, Bellomo R: Renal replacement therapy in acute renal failure. Best Pract Res Clin Anaesthesiol 2004;18:145-157.

17 Aucella F, Di Paolo S, Gesualdo L: Dialysate and replacement fluid composition for CRRT. Contrib Nephrol. Basel, Karger, 2007, vol 156, pp 287-296.

18 Joannidis M, Oudemans-van Straaten H: Clinical review: patency of the circuit in continuous renal replacement therapy. Critical Care 2007; 11:218

19 Hoffart N: Nephrology nursing 1915-1970: a historical study of the integration of technology and care. ANNA J 1989;16:169-178.

20 Coleman B, Merrill JP: The artificial kidney. Am J Nurs 1952;52:327-329.

21 Martin R, Jurschak J: Nursing management of continuous renal replacement therapy. Semin Dialy 1996;9:192-199.

22 Mehta R, Martin R: Initiating and imple menting a continuous renal replacement therapy program. Semin Dial 1996;9:80-87.

23 Baldwin I, Elderkin T: Continuous hemofil tration: nursing perspectives in critical care. New Horizons 1995;3:738-747.

24 Dirkes S, Hodge K: Continuous renal replacement therapy in the Adult Intensive Care Unit. History and current trends. Critical Care Nurse 2007;27:61-80.

25 Canaud B, Leray-Moragues H, Leblanc M, et al: Temporary vascular access for extracorporeal renal replacement therapies in acute renal failure patients. Kidney Int Suppl 1998 66:S142-S150.

26 Schetz M: Vascular access for IHD and CRRT. Contrib Nephrol. Basel, Karger, 2007, vol 156, pp 275-286.

27 Baldwin I: Factors affecting circuit patency and filter life. Contrib Nephrol. Basel, Karger, 2007, vol 156, pp 178-184.
28 Lavaud S, Canivet E, Wuillai A, Maheut H, Randoux C, Bonnet JM, Renaux JL, Chanard $\mathrm{J}$ : Optimal anticoagulation strategy in haemodialysis with heparin coated polyacrylonitrile membrane. Nephrol Dialy Transplant 2003;18:2097-2094.

29 Lavaud S, Paris B, Maheut H, Randoux C, Renaux JL, Rieu P, Chanard J: Assessment of the heparin-binding AN69 ST hemodialysis membrane. II. Clinical studies without heparin administration. ASAIO J 2005;51:348351.

30 Palevsky PM, Baldwin I, Davenport A, Goldstein S, Paganini E: Renal replacement therapy and the kidney: minimising the impact of renal replacement therapy on recovery of acute renal failure. Curr Opin Crit Care 2005; 11:548-554.

31 Paton M: CRRT: help for acute renal failure. Nursing Made Incredibly Easy 2007;5:2838.

32 Mehta RL: Fluid management in continuous renal replacement therapy. Semin Dial 1996; 9:140-144.

33 Fealy N, Baldwin I, Bellomo R: The effect of circuit 'down time' on uraemic control during continuous veno-venous haemofiltration. Crit Care Resus 2002;4:266-270.

$>34$ Davies H, Leslie G: Maintaining CRRT circuit: non-anticoagulant alternatives. Aust Crit Care 2006;19:133-138.

35 Ejaz AA, Komorski RM, Ellis GH, Munjal S: Extracorporeal circuit pressure profiles during continuous venovenous haemofiltration. Nurs Critical Care 2007;12:81-85.

36 Holt A, Bierer P, Bersten A, Bury L, Vedig A: Continuous venovenous haemodiafiltration in critically patients: monitoring circuit function. Anaesth Intens Care 1996;24:423429.

37 Gabutti L, Colucci G, Martella A, Schonholzer C, Marone C: Does monitoring of pre-/ post-dialyzer pressure difference improve efficiency in intermittent hemodialysis? Blood Purif 2003;21:294-300.

>38 Brush KA, Bilodeau ML: Continuous renal replacement therapy. Int Anaesthesiol Clin 2001;39:111-125. 